\title{
Homogeneous cooling and heating states of dilute soft-core gases under nonlinear drag
}

\author{
Satoshi Takada ${ }^{1,2, *}$ \\ ${ }^{1}$ Institute of Engineering, Tokyo University of Agriculture and Technology, 2-24-16 Nakacho, Koganei, Tokyo 184-8588 Japan \\ ${ }^{2}$ Department of Mechanical Systems Engineering, Tokyo University of Agriculture and Technology, 2-24-16 Nakacho, Koganei, Tokyo \\ 184-8588 Japan
}

\begin{abstract}
The temperature evolution of dilute soft inertial gas-solid suspensions is theoretically analyzed when the gas particles are influenced by a nonlinear drag force from a background fluid. The kinetic theory is extended to this system, and the time evolutions of the temperature and the kurtosis of the velocity distribution are derived. Molecular dynamics simulations are also performed to check the validity of the theory, and they show good agreement with the theoretical predictions.
\end{abstract}

\section{Introduction}

Anomalous relaxation processes have recently received much attention from physicists. The Mpemba effect [1], which is an example of this, is known as a process where an initially hotter liquid can be frozen faster than a colder liquid. Similar processes are widely observed in many situations. In particular, the recent theoretical studies on granular gases $[2,3]$ clarified that the appearance of the Mpemba effect in these systems relates to the nonGaussianity of the velocity distribution function (VDF), which exists when the restitution coefficient is smaller than unity. Recently, Santos and Prados [4] considered the system where molecular particles are suspended by a background fluid, where the temperature determined from the kinetic energy of the molecules converges to the environmental temperature, which is determined from the background fluid. They reported that the Mpemba effect appears even in this elastic system when there exists a nonlinear effect of the drag from the background fluid. Their analysis shows that the VDF deviates from the Gaussian in the transient regime, and the magnitude of this deviation determines whether the Mpemba effect appears. However, the comparison of the theory with the simulation is not given in their paper, which means that the applicability of the theory should be clarified.

To this end, we consider the system where soft particles are suspended by the nonlinear background fluid. Recently, the steady-state rheology of this system under shear is theoretically analyzed in terms of the kinetic theory [5], where the softness of particles affects the rheological properties when the shear plays a dominant role with respect to the softness. In this paper, we consider the time evolution of this system under no external forces. We try to extend

*e-mail: takada@go.tuat.ac.jp

A video is available at https://doi.org/10.48448/k3m4-1k49 the theory for the hard-core system to this system, and derive a set of equations that describes the system.

The organization of this paper is as follows: In the next section, we briefly explain our model. Using this information, we extend the kinetic theory to this system in Sec. 3 . The time evolutions of the temperature and the kurtosis of the VDF are derived from the kinetic theory. We solve the time evolution of them from the theory and the simulations in Secs. 4 and 5, respectively. In the last section, we discuss and conclude our results.

\section{Model}

The model of this study is explained in this section. We consider monodisperse particles in the three-dimensional system, where the mass and diameter are $m$ and $\sigma$, respectively. The interaction between particles is given by the harmonic potential [5]

$$
U(r)=\frac{k}{2}(\sigma-r)^{2} \Theta(\sigma-r),
$$

where $r$ is the distance between particles, $k$ relates to the magnitude of the repulsive force, and $\Theta(x)$ is the step function. The scattering process for this potential is needed in the following calculation. The scattering angle $\chi$ is, in general, written by a function of the impact parameter $b$ and the relative speed $v$ between particles. The explicit form of this angle is presented in Ref. [5], which is also used in this paper.

These particles are distributed in the background fluid. If we assume that the effect from the background fluid is simply written by the random force and the kick back interaction, the equation of motion of the particles is described by the Langevin equation

$$
m \frac{d \boldsymbol{v}_{i}}{d t}=\boldsymbol{F}_{i}-\zeta \boldsymbol{v}_{i}+\boldsymbol{\xi}_{i}
$$


We note that this model is a kind of idealized gas-solid suspension model [4-8]. Here, we should determine the form of the drag coefficient $\zeta$. Let us consider a situation where the particles move in a collection of smaller particles (solvent) with the mass $m_{\mathrm{b}}$, the diameter $\sigma_{\mathrm{b}}$, and the density $n_{\mathrm{b}}[4,9]$. The expression of $\zeta$ is derived when we choose a collision model between them. For simplicity, when the collisions between them are assumed to occur via hardcore, the following velocity dependence of $\zeta$ is obtained by solving a scattering problem (see Refs. $[4,9,10]$ ):

$$
\zeta(v)=\frac{2}{3} n_{\mathrm{b}}\left(\sigma+\sigma_{\mathrm{b}}\right)^{2} \frac{\sqrt{2 \pi m_{\mathrm{b}} k_{\mathrm{B}} T_{\mathrm{env}}}}{m+m_{\mathrm{b}}}\left(1+\frac{m_{\mathrm{b}}}{10 m} \frac{m v^{2}}{k_{\mathrm{B}} T_{\text {env }}}\right),
$$

where $T_{\text {env }}$ is the environmental temperature of the smaller particles. Of course, more complicated velocity dependences appear for dense systems. In this paper, however, we consider the system that the nonlinearity appears via the quadratic dependence as above, and for simplicity, we put the form of $\zeta$ given by Eq. (3) as [4, 10]

$$
\zeta(v)=\zeta_{0}\left(1+\gamma \frac{m v^{2}}{k_{\mathrm{B}} T_{\text {env }}}\right)
$$

where $\zeta_{0} \propto T_{\mathrm{env}}^{1 / 2}$, and $\gamma \gtrsim 0$ is assumed to be sufficiently smaller than unity. Therefore, $\boldsymbol{\xi}_{i}$ is the random force which satisfies the following relations [11]:

$\left\langle\boldsymbol{\xi}_{i}(t)\right\rangle=0$,

$\left\langle\xi_{i, \alpha}(t) \xi_{j, \beta}\left(t^{\prime}\right)\right\rangle=2 m \zeta_{0}\left[1+\gamma+\gamma \frac{m v^{2}}{k_{\mathrm{B}} T_{\mathrm{env}}}\right] T_{\mathrm{env}} \delta_{i j} \delta_{\alpha \beta} \delta\left(t-t^{\prime}\right)$

where the bracket $\langle\cdot\rangle$ represents the ensemble average. Here, we introduce the dimensionless parameter $\xi_{\text {env }}$ which characterizes the magnitude of the drag as

$$
\xi_{\mathrm{env}}=\sqrt{\frac{k_{\mathrm{B}} T_{\mathrm{env}}}{m}} \frac{1}{\sigma \zeta_{0}} .
$$

The cooling (heating) process is observed when the initial temperature of the system is higher (lower) than the environmental temperature. In the next section, we derive the equations for the time evolution of the temperature and the non-Gaussianity in terms of the kinetic theory.

\section{Kinetic theory}

Using the above information, we extend the kinetic theory to this system. The Boltzmann equation corresponding to the Langevin equation (2) is given by

$$
\frac{\partial}{\partial t} f(\boldsymbol{v})-\frac{\partial}{\partial \boldsymbol{v}} \cdot\left[\zeta(v)\left(\boldsymbol{v}+\frac{k_{\mathrm{B}} T_{\text {env }}}{m}\right) f(\boldsymbol{v})\right]=J[\boldsymbol{v} \mid f, f],
$$

where $f(\boldsymbol{v})$ is the VDF and $J[\boldsymbol{v} \mid f, f]$ is the collision integral given by $[6-8,12]$

$$
\begin{aligned}
& J\left[\boldsymbol{v}_{1} \mid f, f\right]=\int d \boldsymbol{v}_{2} \int d \hat{\boldsymbol{k}} \Theta(\sigma-b)\left|\boldsymbol{v}_{12} \cdot \hat{\boldsymbol{k}}\right| \\
& \times\left[\sigma_{\mathrm{s}}\left(\chi, v_{12}^{\prime}\right) f\left(\boldsymbol{v}_{1}^{\prime}\right) f\left(\boldsymbol{v}_{2}^{\prime}\right)-\sigma_{\mathrm{s}}\left(\chi, v_{12}\right) f\left(\boldsymbol{v}_{1}\right) f(\boldsymbol{v})\right],
\end{aligned}
$$

where $\boldsymbol{v}_{12} \equiv \boldsymbol{v}_{1}-\boldsymbol{v}_{2}$ is the relative velocity, $\sigma_{\mathrm{s}}$ is the scattering cross section which is determined from the scattering angle $\chi$, and $\left(\boldsymbol{v}_{1}^{\prime}, \boldsymbol{v}_{2}^{\prime}\right)$ are the post-collisional velocities satisfying

$$
\boldsymbol{v}_{1}^{\prime}=\boldsymbol{v}_{1}-\left(\boldsymbol{v}_{12} \cdot \hat{\boldsymbol{k}}\right) \hat{\boldsymbol{k}}, \quad \boldsymbol{v}_{2}^{\prime}=\boldsymbol{v}_{2}+\left(\boldsymbol{v}_{12} \cdot \hat{\boldsymbol{k}}\right) \hat{\boldsymbol{k}}
$$

We note that the softness of the particles appears in the collision integral (8).

By considering the second moment of Eq. (7) with respect to the velocity, the temperature evolution is given by [4]

$$
\frac{d T}{d t}=-2 \zeta_{0}\left(T-T_{\text {env }}\right)\left(1+5 \gamma \frac{T}{T_{\text {env }}}\right)-10 \zeta_{0} \frac{T^{2}}{T_{\text {env }}} a_{2} .
$$

Here, we have introduced the kurtosis of the VDF as

$$
a_{2}=\frac{3}{5} \frac{\left\langle v^{4}\right\rangle}{\left\langle v^{2}\right\rangle^{2}}-1
$$

This is because the VDF deviates from the Gaussian when the temperature evolves [4].

Let us expand the dimensionless VDF $\tilde{f} \equiv\left(v_{\mathrm{T}}^{3} / n\right) f(\boldsymbol{v})$ in terms of the Sonine polynomials [4]

$$
\tilde{f}(\boldsymbol{c})=\pi^{-3 / 2} \exp \left(-c^{2}\right)\left[1+a_{2}\left(\frac{1}{2} c^{4}-\frac{5}{2} c^{2}+\frac{15}{8}\right)\right],
$$

with the thermal velocity $v_{\mathrm{T}} \equiv \sqrt{2 T / m}$ and the dimensionless velocity $\boldsymbol{c} \equiv \boldsymbol{v} / v_{\mathrm{T}}$, where we only consider the lowest order of $a_{2}$ in Eq. (12). It is noted that this coefficient $a_{2}$ is consistent with Eq. (11). We assume that the coefficient $a_{2}$ is sufficiently small with respect to unity, which will be checked later.

Using the dimensionless form of the VDF and the Sonine expansion (12), Eq. (7) can be rewritten as

$$
\begin{aligned}
& \frac{\partial}{\partial t} \tilde{f}(\boldsymbol{c})-\frac{\partial}{\partial \boldsymbol{c}} \cdot\left[\frac{1}{2 T} \frac{\partial T}{\partial t} \boldsymbol{c}+\zeta_{0}\left(1+\gamma \frac{2 T}{T_{\mathrm{env}}} c^{2}\right)\right. \\
& \left.\quad \times\left(\boldsymbol{c}+\frac{T_{\mathrm{env}}}{2 T} \frac{\partial}{\partial \boldsymbol{c}}\right)\right] \tilde{f}(\boldsymbol{c})=v_{\mathrm{env}} \sqrt{\frac{T}{T_{\mathrm{env}}}} \tilde{J}[\boldsymbol{c} \mid \tilde{f}, \tilde{f}]
\end{aligned}
$$

with the collision frequency

$$
v_{\mathrm{env}}=n \sigma^{2} \sqrt{\frac{2 k_{\mathrm{B}} T_{\mathrm{env}}}{m}} .
$$

Here, $n$ is the number density of the system.

For further calculation, let us introduce the fourth moment of the collision integral $\mu_{4}$ [13]:

$$
\mu_{4}=\frac{\sqrt{2 \pi}}{4} a_{2} \int_{0}^{\infty} d c \int_{0}^{\infty} d b^{*} b^{*} c^{7} \sin ^{2} \chi \exp \left(-\frac{c^{2}}{2}\right)
$$

with $b^{*} \equiv b / \sigma$. We note that the fourth collisional moment $\mu_{4}$ relates to the Omega integral $[5,12]$

$$
\Omega_{k, l}(T)=\sqrt{\frac{k_{\mathrm{B}} T}{\pi m}} \int_{0}^{\infty} d y e^{-y^{2}} y^{2 k+3} Q_{l}\left(2 y \sqrt{\frac{k_{\mathrm{B}} T}{m}}\right),
$$

as

$$
\mu_{4}\left(T^{*}\right)=4 \sqrt{2 \pi} a_{2} \frac{\Omega_{2,2}(T)}{\Omega_{2,2}^{\mathrm{HC}}(T)} \equiv 4 \sqrt{2 \pi} a_{2} \Omega_{2,2}^{*}\left(T^{*}\right),
$$


where $Q_{l}(v)$ is defined by

$$
Q_{l}(v)=2 \pi \int_{0}^{\infty} d b b\left[1-\cos ^{l} \chi(b, v)\right] .
$$

Here, $T^{*}=k_{\mathrm{B}} T /\left(k \sigma^{2}\right)$ is the dimensionless temperature, and $\Omega_{2,2}^{\mathrm{HC}}(T)=2 \sigma^{2}\left(\pi k_{\mathrm{B}} T / m\right)^{1 / 2}$ is the hard-core limit of $\Omega_{2,2}(T)$. We note that $\Omega_{2,2}^{*}\left(T^{*}\right)$ behaves as $1-\Omega_{2,2}^{*}\left(T^{*}\right) \propto$

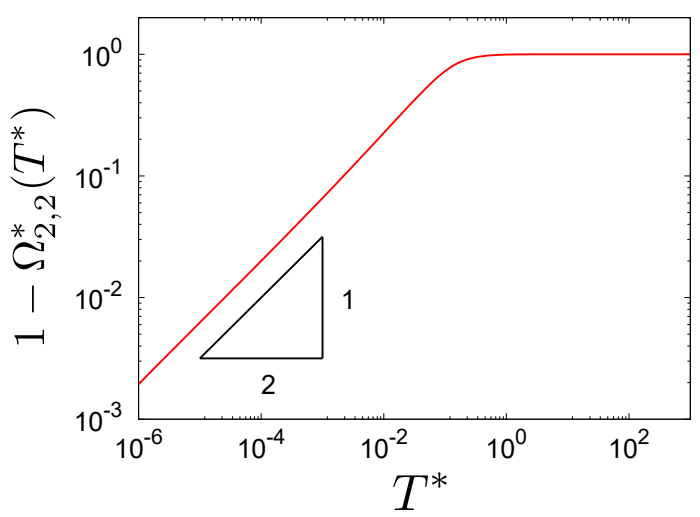

Figure 1. Temperature dependence of the dimensionless Omega integral $\Omega_{2,2}^{*}\left(T^{*}\right)$, where we have introduced $T^{*} \equiv k_{\mathrm{B}} T /\left(k \sigma^{2}\right)$ and $\Omega_{2,2}^{*}\left(T^{*}\right) \equiv \Omega_{2,2}(T) / \Omega_{2,2}^{\mathrm{HC}}(T)$. The triangle represents the slope of $1-\Omega_{2,2}^{*}\left(T^{*}\right)$ in the low temperature regime.

$T^{* 1 / 2}$ and $\Omega_{2,2}^{*}\left(T^{*}\right) \sim T^{*-2}$ in the low and high temperature regimes, respectively, as shown in Fig. 1 (see also Fig. 5 of Ref. [5]). For practical calculations, we prepare a numerical table of $\Omega_{2,2}^{*}\left(T^{*}\right)$ for $k_{\mathrm{B}} T /\left(k \sigma^{2}\right)=10^{0.05 n}(n$ is an integer) in the range $10^{-10} \leq k_{\mathrm{B}} T /\left(k \sigma^{2}\right) \leq 10^{8}$ and interpolate the value for any $T$.

\section{Homogeneous cooling and heating states}

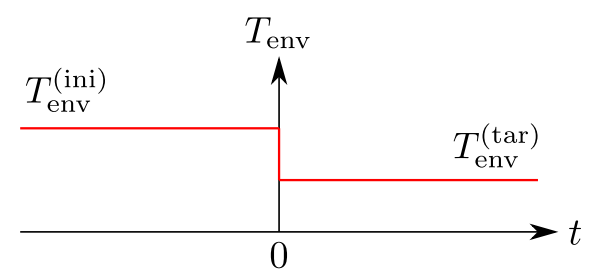

Figure 2. Schematic of the protocol. The environmental temperature is set as $T_{\mathrm{env}}=T_{\mathrm{env}}^{\text {(ini) }}$ for $t<0$, and is changed to $T_{\mathrm{env}}=T_{\mathrm{env}}^{\text {(tar) }}$ at $t=0$. Here, the case for $T_{\mathrm{env}}^{(\mathrm{ini})}>T_{\mathrm{env}}^{(\mathrm{tar})}$ is shown.

In this section, let us solve the time evolution of the system. So far, we assume that the environmental temperature is fixed. However, we can control the value of the environmental temperature at $t=0$ as shown in Fig. 2. We introduce two environmental temperatures $T_{\text {env }}^{\text {(ini) }}$ and $T_{\mathrm{env}}^{\text {(tar) }}$, which correspond to $t<0$ and $t \geq 0$, respectively. Here, the system is in equilibrium with the initial parameter $\xi_{\text {env }}^{\text {(ini) }}=\left(k_{\mathrm{B}} T_{\text {env }}^{(\text {ini })} / m\right)^{1 / 2} /\left(\sigma \zeta_{0}\right)$ for $t<0$, and we discontinuously change the value of $\xi_{\text {env }}$ to $\xi_{\text {env }}^{(\text {tar })}=$ $\left(k_{\mathrm{B}} T_{\mathrm{env}}^{(\mathrm{tar})} / m\right)^{1 / 2} /\left(\sigma \zeta_{0}\right)$ at $t=0$. Here, we choose the values of the softness as $k^{*} \equiv k /\left(m \zeta_{0}^{2}\right)=10^{4}, 10^{0}$, and $10^{-2}$. In this paper, we fix $\xi_{\text {env }}^{(\mathrm{tar})}=1.0$ and $\gamma=0.1$ for simplicity.

To describe the dynamics of the system, let us introduce the following dimensionless parameters:

$$
\theta \equiv \frac{T}{T_{\mathrm{env}}^{(\mathrm{tar})}}, \quad \theta_{0} \equiv \frac{T_{\mathrm{env}}^{(\mathrm{ini})}}{T_{\mathrm{env}}^{(\mathrm{tar})}}, \quad \tau \equiv \zeta_{0} t .
$$

From Eqs. (10) and (13), we obtain a set of equations as

$$
\begin{aligned}
\frac{d \theta}{d \tau}=-2(\theta-1)(1+ & 5 \gamma \theta)-10 \theta^{2} a_{2} \\
\frac{d a_{2}}{d \tau}=-8 \gamma(\theta-1)- & {\left[\frac{4}{\theta}-8 \gamma+44 \gamma \theta\right.} \\
& \left.+\frac{64}{5 \sqrt{\pi}} \varphi \xi_{\text {env }} \sqrt{\theta} \Omega_{2,2}^{*}\left(\frac{\theta}{k^{*}}\right)\right] a_{2} .
\end{aligned}
$$

This set of equations is equivalent to that reported in Ref. [4] when we consider the hard-core limit. We note that the expression of the time evolution of the temperature (20a) is the same with that for hard-core gases, which is because the symmetric property of the second moment of the collision integral is unchanged even when we consider the soft-core gases. This means that the softness affects the system only via the evolution of the non-Gaussianity $a_{2}$ (20b). If we can ignore $a_{2}$ in Eq. (20a), the time evolution of the dimensionless temperature is explicitly given by

$$
\theta_{a_{2}=0}(\tau)=1+\frac{(1+5 \gamma)\left(\theta_{0}-1\right)}{\left(1+5 \gamma \theta_{0}\right) e^{2(1+5 \gamma) \tau}-5 \gamma\left(\theta_{0}-1\right)} .
$$

It should be noted that, as explained in the above, this expression is independent of the potential (1).

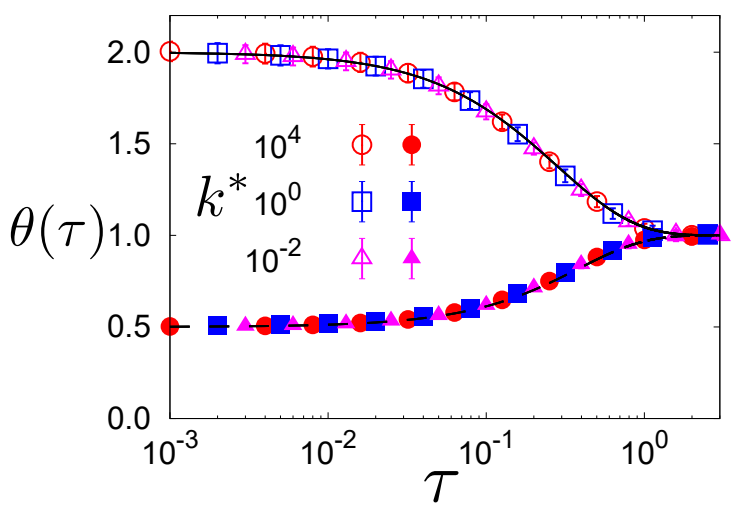

Figure 3. Time evolutions of the dimensionless temperature from the theory (20a) for $\theta_{0}=2$ (solid line) and 0.5 (dashed line) when we choose the softness of the particles as $k^{*} \equiv k /\left(m \zeta_{0}^{2}\right)=$ $10^{4}, 10^{0}$, and $10^{-2}$. Here, the difference between lines is almost invisible. The symbols represent the simulation results. We note that the error bars are smaller than the symbols.

Let us solve the set of equations (20) with the initial condition $\theta(0)=\theta_{0}$. It should be noted that $a_{2}(0)=0$ is satisfied because the system is in equilibrium for $t<0$. Figures 3 and 4 show the time evolutions of $\theta$ and $a_{2}$ with $\theta_{0}=2$ and 0.5 , respectively. We find that the time evolutions are almost independent of the softness of the particles. We also find that the transient process of the temperature is monotonic, independent of cooling and heating processes. Here, the magnitude of $a_{2}$ is always smaller than 


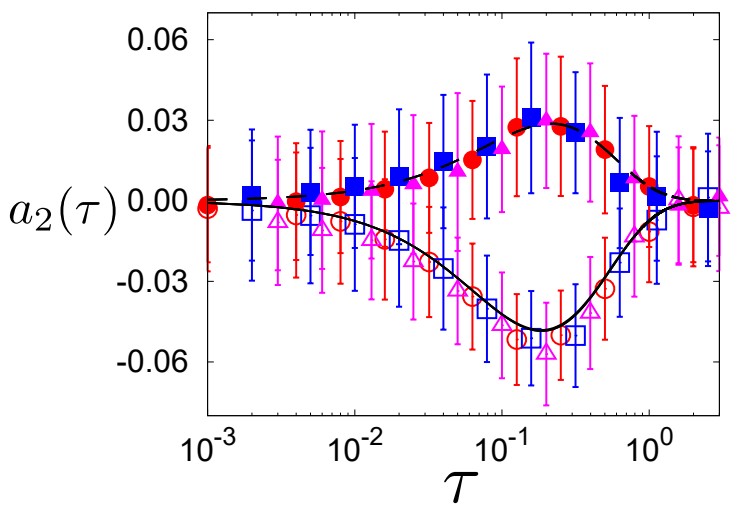

Figure 4. Time evolutions of the kurtosis obtained from the theory (20b) for $\theta_{0}=2$ (solid line) and 0.5 (dashed line) when we choose the softness of the particles as $k^{*} \equiv k /\left(m \zeta_{0}^{2}\right)=10^{4}, 10^{0}$, and $10^{-2}$. Here, the difference between lines is almost invisible. The symbols represent the simulation results. The lines and symbols are the same with those in Fig. 3.

unity, which is consistent with the previous assumption. We note that the sign of $a_{2}$ in the initial stage is negative (positive) for the cooling (heating) process. This can be easily understood by Eq. (20b). Here, the sign of the first term is determined whether the dimensionless temperature is larger or smaller than unity.

\section{Molecular dynamics simulations}

In this section, let us check the validity of the theory by performing the molecular dynamics simulations [11]. We prepare $N=10^{3}$ particles in the cubic box, where the linear length of the system is fixed as $L=37.1 \sigma$. Here, the correspondingly packing fraction becomes $\varphi(=$ $\left.(\pi / 6) N \sigma^{3} / L^{3}\right)=1.0 \times 10^{-2}$. We also adopt the periodic boundary condition in all directions. We numerically solve the equation of motion (2) for each particle with the dimensionless time increment $\Delta t^{*}=\zeta_{0} \Delta t=1.0 \times 10^{-5}$, which is small enough for the convergence of the results. We use 100 different initial conditions to calculate the ensemble average of the results. Here, the kurtosis is evaluated in terms of the definition (11).

As shown in Figs. 3 and 4, the simulation results well reproduce the theoretical prediction of the time evolutions of the dimensionless temperature and the kurtosis of the VDF. Even in the simulations, the measured value of $a_{2}$ is much smaller than unity, which suggests the validity of the assumption used to derive the set of equations. We also note that the dependence of the evolutions on the softness is small in the simulations.

\section{Discussion and Conclusion}

In this paper, the system consists of the soft particles whose interparticle interaction is given by Eq. (1). As explained previously, the equation for the time evolution of the temperature (20a) is the same as that for the hard-core system reported in Ref. [4]. The difference between hard and soft-core systems appears only from $a_{2}$, but this effect is sufficiently small because the magnitude of $a_{2}$ is smaller than unity as shown in Fig. 4.

The Mpemba effect is observed when one controls the initial non-Gaussianity $a_{2}$ in the hard-core limit [4]. To check the realization of this in our model is important, but this is our future work. There, we should consider how we control the initial non-Gaussianity $a_{2}$ is not simple in experiments or simulations.

In this paper, we have investigated the time evolution of the temperature when molecular gases are influenced by the nonlinear drag. We have derived the evolutions of the temperature and the non-Gaussianity of the velocity distribution function from the kinetic theory. We have also performed the molecular dynamics simulations, and we have confirmed that the numerical results are well reproduced by those from the kinetic theory for the wide range of the softness of the particles.

\section{Acknowledgment}

The author thanks Haruto Ishikawa for his kind support. This work is supported by the Grant-in-Aid of MEXT for Scientific Research (Grant No. JP20K14428).

\section{References}

[1] E.B. Mpemba, D.G. Osborne, Phys. Educ. 4, 172 (1969)

[2] A. Lasanta, F.V. Reyes, A. Prados, A. Santos, Phys. Rev. Lett. 119, 148001 (2017)

[3] A. Torrente, M.A. López-Castaño, A. Lasanta, F. Vega Reyes, A. Prados, A. Santos, Phys. Rev. E 99, 060901(R) (2019)

[4] A. Santos, A. Prados, Phys. Fluids 32, 072010 (2020)

[5] S. Sugimoto, S. Takada, J. Phys. Soc. Jpn. 89, 084803 (2020), J. Phys. Soc. Jpn. 89, 127001 (2020) (addendum)

[6] H. Hayakawa, S. Takada, Prog. Theor. Exp. Phys. 2019, 083J01 (2019)

[7] H. Hayakawa, S. Takada, V. Garzó, Phys. Rev. E 96, 042903 (2017), Phys. Rev. E 101, 069904 (2020) (erratum)

[8] S. Takada, H. Hayakawa, A. Santos, V. Garzó, Phys. Rev. E 102, 022907 (2020)

[9] M. Hohmann, F. Kindermann, T. Lausch, D. Mayer, F. Schmidt, E. Lutz, A. Widera, Phys. Rev. Lett. 118, 263401 (2017)

[10] L. Ferrari, Chem. Phys. 336, 27 (2007)

[11] H. Risken, The Fokker-Planck equation: methods of solution and applications (Springer, Berlin, 1996)

[12] S. Chapman, T.G. Cowling, The mathematical theory of nonuniform gases (Cambridge University Press, New York, 1970)

[13] S. Takada, K. Saitoh, H. Hayakawa, Phys. Rev. E 94, 012906 (2016) 